\title{
3D detection and quantitative characterization of cracks in a ceramic matrix composite tube using X-ray computed tomography
}

Y. Chen ${ }^{1,2, *}$, L. Gélébart ${ }^{1}$, C. Chateau ${ }^{2}$, M. Bornert ${ }^{2}$, A. King ${ }^{3}$, P. Aimedieu ${ }^{2}$, C. Sauder $^{1}$

'DEN-Service de Recherches Métallurgiques Appliquées, CEA, Université ParisSaclay, F-91191, Gif-sur-Yvette, France

²Laboratoire Navier (UMR 8205), CNRS, ENPC, IFSTTAR, Université Paris-Est, F77455 Marne-la-Vallée, France

${ }^{3}$ Synchrotron SOLEIL, St-Aubin 91192, France

\begin{abstract}
Cracks play an essential role in the degradation of the thermomechanical behavior of ceramic matrix composites. However, characterizing their complex 3D geometries within a complex microstructure is still a challenge. This paper presents a series of procedures, based on X-ray tomographic images, to evaluate the applied 3D strains, including their through-thickness gradients, and to detect and quantify the induced crack networks in ceramic matrix composites. Digital volume correlation and some dedicated image processing algorithms are employed. A novel method is proposed to estimate the opening, orientation and surface area of the detected cracks. The proposed procedures are applied to the images of a $\mathrm{SiC} / \mathrm{SiC}$ composite tube that has been tested in situ under uniaxial tension with synchrotron X-ray computed tomography.
\end{abstract}

Keywords: Ceramic matrix composites (CMCs); Damage mechanisms; X-ray computed tomography; Crack quantification

NOTICE: This is a post-peer-review, pre-copyedit version of an article published in Experimental Mechanics. The final authenticated version is available online at: https://doi.org/10.1007/s11340-019-00557-5.

*Corresponding author, Email: chenyangpic@gmail.com 


\section{Introduction}

Because of their outstanding physical and chemical properties at high temperatures in comparison with metals, silicon carbide $(\mathrm{SiC})$ composite materials are studied as fuel cladding materials either for future advanced fission/fusion reactors [1] or, more recently, for the currently existing light water reactors [2]. SiC/SiC composites, manufactured by chemical vapor infiltration (CVI) process, exhibit nonlinear damageable mechanical behavior governed by microcracking within the material (see e.g. $[3,4])$. Optimal structural design of such ceramic matrix composites (CMCs) requires a good understanding of the relationships between the microstructure, the damage mechanisms and the macroscopic behavior.

Due to their complex microstructures, it is necessary to investigate CMCs by collecting 3D, spatially resolved information. Acoustic emission and electric resistance are two commonly used techniques for this purpose [5-8]. However, they cannot provide explicit information about the growth path and the nature of local fractures. X-ray computed tomography (XRCT) has been proven to be a powerful tool for studying this category of materials [9-11], because it enables an explicit and direct observation of the 3D geometry and growth path of cracks, which are important for understanding the damage behavior of CMCs. Both qualitative and quantitative investigations of damage mechanisms have been conducted using in situ XRCT for unidirectional minicomposites loaded axially [12-14]. However, it is still a challenge to extend this method, even when applied to simple specimen geometry, to more realistic architectured textile composites because of the large size of the volumes to be considered. On the one hand, such volumes are required to encompass several unit cells of the quasi-periodic woven microstructure. On the other hand, they must resolve both its smaller features, such as micropores, and the induced complex crack networks, which may exhibit rather small openings.

The Digital Volume Correlation (DVC) technique has already been coupled with in situ XRCT in order to quantify local or global deformation of CMCs under load (see e.g. $[15,16])$. The DVC-evaluated displacements can be used to measure the crack opening displacement (COD) [17]. The 3D strain field can also be a relevant indicator of damage [18]. However, this method is limited by the accuracy and spatial resolution of the DVC-evaluated displacement field, especially in the case of $\mathrm{SiC} / \mathrm{SiC}$ composites, for which the image contrast of the microstructure is not sufficiently local nor uniformly distributed, because it mostly arises from residual micropores, elongated and aligned along the tow direction. Furthermore, extracting quantitative information about both the 3D microstructure and damage requires sophisticated image processing algorithms, the application of which may take much more time than the experiment itself. As a result, many applications of such approach are limited to brief qualitative observations of only a few regions of interest of limited size. However, an optimal use of the huge amount of numerical data provided by such in situ XRCT experiments, as well as of the long and thorough efforts developed to produce them, would of course imply quantitative processing of all available data. In addition, because of the natural statistical fluctuations of damage events within a microstructure, the imaging and associated analyses should be performed over sufficiently large domains to ensure statistical representativeness.

Cracks in CMCs are numerous and usually organized in networks with complex 3D geometries, which spread over the complex porous microstructure of the composite. 
Moreover, the opening of cracks is usually very small, typically below a few micrometers in the case of $\mathrm{SiC} / \mathrm{SiC}$ composites [19]. Therefore, detecting them in the whole field of view is not straightforward and requires automated and sensitive procedures. For such small openings of cracks, artifacts commonly observed in tomographic images may hinder the crack detection and need to be treated carefully. Inherently, tomographic observations will never be able to capture all damage events in the material, and crack detection will be essentially limited by image resolution, or voxel size. Nevertheless, a subvoxel detectability, i.e. a capability to detect cracks with an opening below one voxel, can be expected by virtue of the information carried by the image gray levels [20].

The objective of this paper is to introduce a series of new procedures to fully analyze 3D tomographic images, in order to (i) evaluate the overall strain of a tubular sample, including its through-thickness heterogeneity; (ii) detect complex crack networks; (iii) measure their local opening, orientation and surface area of cracks, and average them over representative domains. These procedures are here used to analyze the damage mechanisms in a $\mathrm{SiC} / \mathrm{SiC}$ composite tube under in situ tensile load on a synchrotron XRCT platform. After a brief description of the studied material and the experimental setup in section 2, we present the post processing of the tomographic images in section 3. Finally, some results are illustrated in section 4.

\section{Material and Methods}

\subsection{Material}

The studied material is a $\mathrm{SiC} / \mathrm{SiC}$ composite tube based on $3^{\text {rd }}$ generation $\mathrm{SiC}$ fibers (Hi-Nicalon type S) of $12 \mu \mathrm{m}$ average diameter. The fibers have been coated with a $30 \mathrm{~nm}$ pyrocarbon interphase and the fiber preform has been densified by $\mathrm{SiC}$ matrix, both using the Chemical Vapor Infiltration (CVI) process. The fiber architecture is composed of two layers of $2 \times 22 \mathrm{D}$ braided tows (Fig.1.a), which are oriented at $\pm 45^{\circ}$ with respect to the tube axis. The inner surface of the tube has been ground after densification to be smooth, so as to simulate the real fuel cladding concept for the nuclear application. In order to improve the spatial resolution of tomographic images, the sample needs to be as small as possible, yet the tubular geometry should ideally be kept to avoid boundary effects induced by cut edges. Therefore, the tube dimension has been reduced to a regular (ground) internal diameter of $3.47 \mathrm{~mm}$ and a rough external diameter of $\sim 4.90 \mathrm{~mm}$, instead of $7.85 \mathrm{~mm}$ and $\sim 9.6 \mathrm{~mm}$ respectively for the real industrial application.

\subsection{In situ tomographic images}

The in situ tensile test has been performed at the French synchrotron facility SOLEIL on the PSICHE beamline [21]. The in-situ loading device uses an aluminum tube with an external diameter of $20 \mathrm{~mm}$ and a wall thickness of $2 \mathrm{~mm}$ to transfer the loading force from the uniaxial actuator to the sample (Fig.1.b and c).

A polychromatic $\mathrm{X}$-ray beam with a pink spectrum centered at $45 \mathrm{keV}$ passes through the aluminum tube and the sample, and is captured by the detector system that is composed of a scintillator, a $45^{\circ}$ mirror, a $2.5 x$ optic lens and a Hamamatsu Orca 
Flash4.0 camera, based on a $2048 \times 2048$ CMOS sensor with a pixel size of $6.5 \mu \mathrm{m}$. One rotation of $360^{\circ}$ (6000 projections per rotation) has been performed for each scan. The image acquisitions have been carried out at one reference unloaded state and then at five uniaxial tension levels. In order to investigate a larger vertical field of view, two successive scans have been acquired for each loading level at different axial positions, with an overlap of $0.72 \mathrm{~mm}$ along the axial direction.
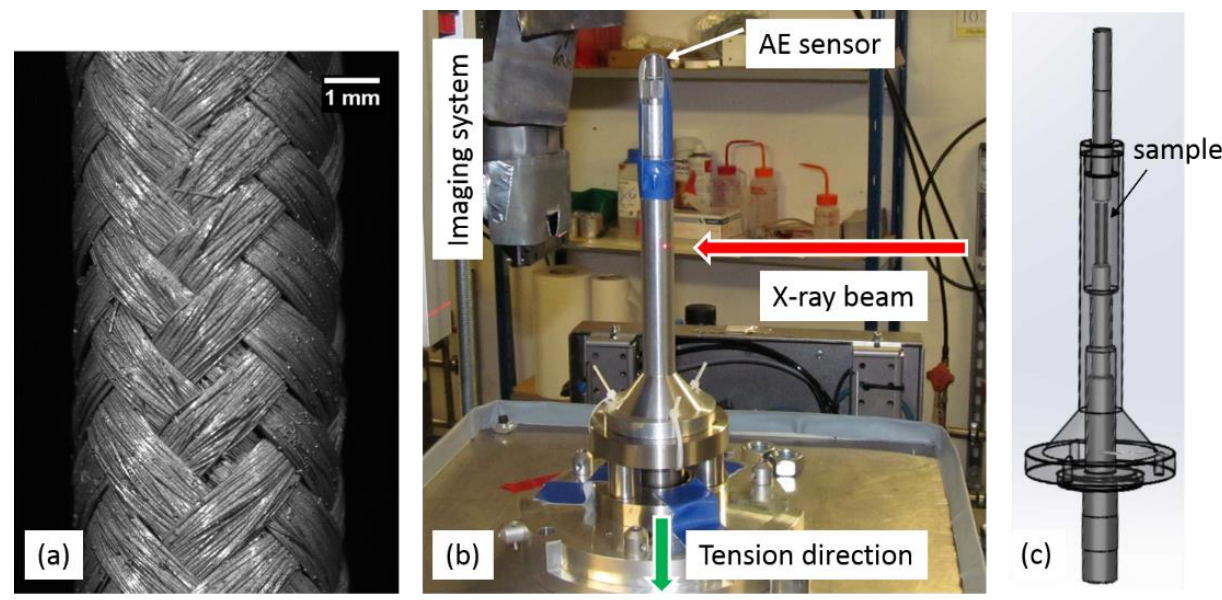

Fig. 1 (a) View of the outer surface of a 2D-braided SiC/SiC composite tube. (b) Experimental setup at the PSICHE beamline of SOLEIL Synchrotron. (c) Sketch of the loading device.

The 3D images are reconstructed using the software PyHST2 [22], and converted into 8 bit unsigned integer format. Each reconstructed image has a dimension of $2048 \times 2048 \times 1024$ voxels, corresponding to $5.3 \times 5.3 \times 2.7 \mathrm{~mm}^{3}$ with a voxel size of 2.6 $\mu \mathrm{m}$. A cross-sectional slice is shown in Fig.2.a. Even though the voxel size is smaller than the fiber diameter $(\sim 12 \mu \mathrm{m})$, the fiber and the matrix can barely be distinguished because of their low density contrast: CVI SiC and Hi-Nicalon type S SiC fibers are both close to stoichiometric SiC. Macro- and micropores can however be clearly seen and distinguished. The image artifacts are highlighted by a gray-level profile across the tube thickness in Fig.2.b. They need to be carefully treated during the crack detection procedure. Fig. 3 shows that both the fiber architecture and cracks can be observed in the 3D image. Extracting such rich information requires specific techniques that will be introduced in the next section. 

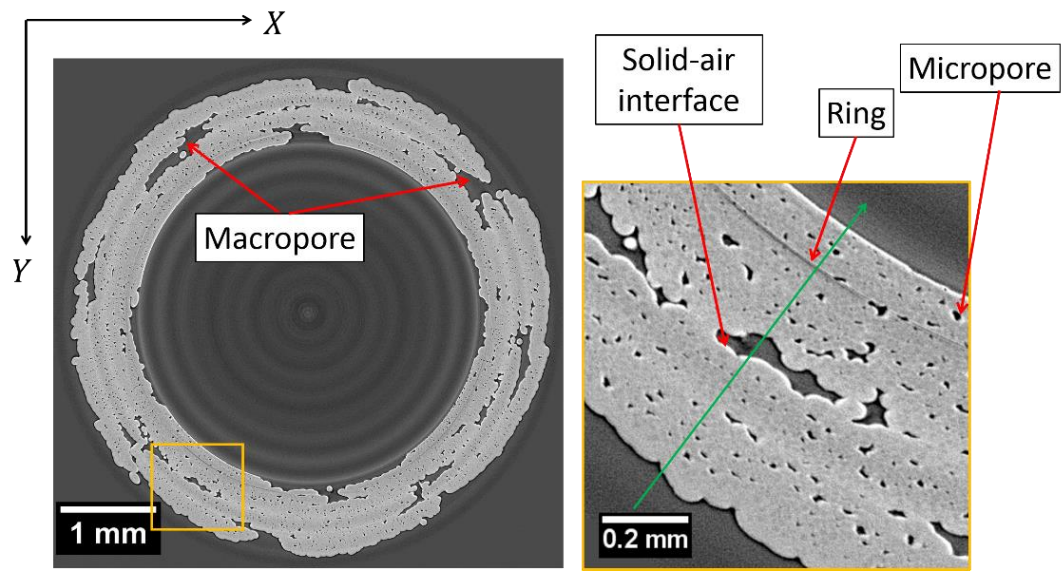

(a)

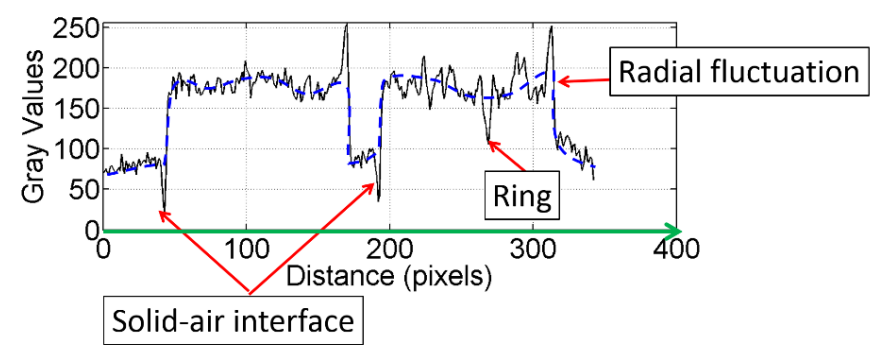

(b)

Fig.2 Reconstructed image: (a) cross-sectional slice and a zoom; (b) Gray-level profile along the green line in the cross-sectional slice in (a).

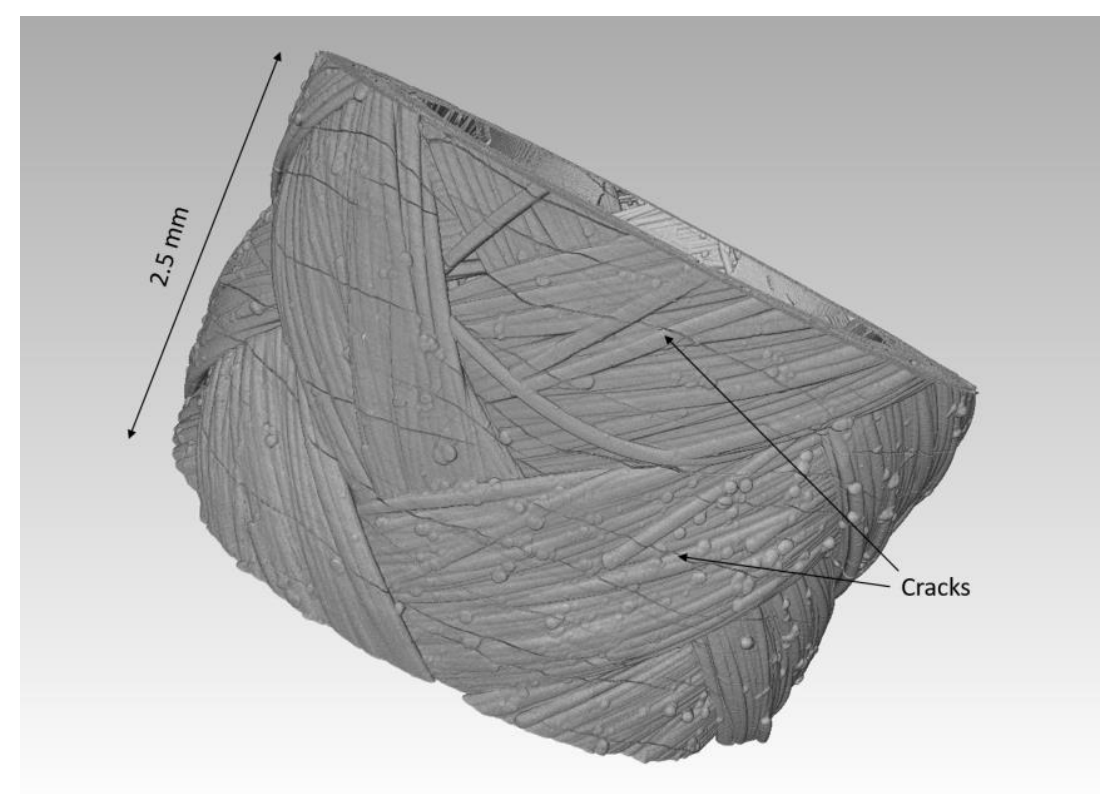

Fig. 33 volume rendering of one of the two sequential volumes at the last loading step. 


\section{Image processing}

First of all, an in-house DVC software CMV3D [23] using local cross correlation is employed to evaluate the displacement fields. The spatial resolution of the latter is linked to the available local image contrast, essentially provided by micro and macropores. The DVC calculations are performed over the two sequential images separately (i.e. without being stitched). In order to minimize the computation time, an idealized tube geometry has been determined from the 3D images, and the correlation grids are defined in cylindrical coordinates only over the tube thickness region. Cubic correlation window with variable size has been used: 30 voxels per side for an initial attempt, increased to 50 voxels per side when the optimal correlation coefficient turns out to be larger than a threshold of 0.3. More details about the DVC procedure can be found in [24].

Based on the DVC results, novel procedures are proposed to fully analyze the tomographic data. The average tensile, radial, circumferential and torsional strains of the composite tube, including their through-thickness heterogeneities will be evaluated from the displacement measurements resulting from the two separate DVC analyses of the volume sets. Cracks will be detected and quantified using a method making full use of image gray levels.

\subsection{Strain evaluation}

The contrast within the $3 \mathrm{D}$ images is provided by micro- and macropores. The micropores provide rather local features, but in a limited number. Moreover, they are very elongated and aligned along the direction of the tow they belong to. As a result, this kind of contrast is not very suitable for a precise and detailed evaluation of the local displacement field. Combined with the small deformability of the ceramic constituents, the local strains as deduced from the DVC-measured displacement field at the scale of the grid spacing, are very noisy. It must be emphasized that the displacement field in the present study is not sufficiently spatially resolved, nor accurate to evaluate crack openings from kinematic discontinuities evaluated this way, and an alternative idea for crack quantification will be proposed in section 3.3.

Hence, we introduce here a method for measuring profiles throughout the tube thickness of the strains averaged over the tube length and circumference. Note first that the set of correlation points at the same radial position defines a radial (cylindrical) layer $I$. Their displacements are fitted to those of a uniformly deformed cylinder that can be arbitrarily translated along and rotated around the tube axis. The uniform deformation of a cylindrical layer $I$ is described by its axial strain $\varepsilon_{z z}^{I}$, radial displacement $u_{r}^{I}$ and torsion angle per unit length $\lambda^{I}$ with respect to the tube axis:

$$
\left(\begin{array}{l}
r_{0} \\
\theta_{0} \\
z_{0}
\end{array}\right) \rightarrow\left(\begin{array}{l}
r \\
\theta \\
z
\end{array}\right)=\left(\begin{array}{c}
r_{0}+u_{r}^{I} \\
\theta_{0}+\lambda^{I} \cdot z_{0}+\beta_{z}^{I} \\
\left(1+\varepsilon_{z z}^{I}\right) \cdot z_{0}+T_{z}^{I}
\end{array}\right)
$$

where $\left[r_{0}, \theta_{0}, z_{0}\right]^{t}$ and $[r, \theta, z]^{t}$ respectively represent the position before and after the transformation of a material point, with respect to the tube-cylindrical coordinate system. $T_{z}^{I}$ and $\beta_{z}^{I}$ stand for the axial translation along and rotation around the tube axis of the considered layer $I$. 
The initial and deformed positions are transformed from the tube-cylindrical to the image-Cartesian coordinates:

$$
\left(\begin{array}{c}
X_{0}^{K} \\
Y_{0}^{K} \\
Z_{0}^{K}
\end{array}\right)=\boldsymbol{R}_{\mathbf{0}}^{K} \cdot\left(\begin{array}{c}
r_{0} \cdot \cos \theta_{0} \\
r_{0} \cdot \sin \theta_{0} \\
Z_{0}
\end{array}\right)+\underline{t}_{0}^{K} \text { and }\left(\begin{array}{c}
X^{K} \\
Y^{K} \\
Z^{K}
\end{array}\right)=\boldsymbol{R}^{K} \cdot\left(\begin{array}{c}
r \cdot \cos \theta \\
r \cdot \sin \theta \\
z
\end{array}\right)+\underline{t}^{K}
$$

where the superscript $*^{K} \quad(K=1,2)$ denotes the quantity $*$ in one of the two sequential images. $\boldsymbol{R}_{0}^{K}$ and $\underline{t}_{0}^{K}$ are the rotation matrix and translation vector describing the coordinate transformation (from tube to image coordinates) in the reference configuration. They can be determined when identifying the tube axis (see [24], Chapter 2.2.1), and are identical for the two sequential images at different axial positions $\left(\boldsymbol{R}_{\mathbf{0}}^{1}=\boldsymbol{R}_{\mathbf{0}}^{2} ; \underline{t}_{0}^{1}=\underline{t}_{0}^{2}\right)$. Similarly, $\boldsymbol{R}^{K}$ and $\underline{t}^{K}$ describe the coordinate transformation in the deformed configuration. Yet, they are allowed to be different for the two sequential images, which makes it possible to take into account the potential difference in coordinate change between the two sequential volumes after mechanical transformation. In fact, this difference could arise if the sample is not exactly repositioned between the two scans. As a result, $\boldsymbol{R}^{K}$ and $\underline{t}^{K}$ are six parameters for each volume ( 3 rotation angles and 3 translation components) to be determined by the procedure of transformation fitting, together with the five parameters per radial layer $\left(\varepsilon_{z z}^{I}, u_{r}^{I}, \lambda^{I}, T_{z}^{I}\right.$ and $\left.\beta_{z}^{I}\right)$.

The fitting procedure is completed through a least-square minimization procedure (Isqcurvefit function in MATLAB) between the simulated displacement field $\underline{u}^{\text {sim,K }}$ and the DVC-measured one $\underline{u}^{D V C, K}$ :

$$
\alpha^{o p t}=\arg \min _{\alpha} \sum_{K=1}^{2} \sum_{I=1}^{m} \sum_{j=1}^{n}\left\|\underline{u}^{\operatorname{sim}, K}\left(\underline{X}_{j}^{I}, \alpha\right)-\underline{u}^{D V C, K}\left(\underline{X}_{j}^{I}\right)\right\|
$$

where $\alpha=\left\{\left\{\varepsilon_{z z}^{I}, u_{r}^{I}, \lambda^{I}, \beta_{z}^{I}, T_{z}^{I}\right\}_{I=1,2, \ldots, m} ;\left\{\boldsymbol{R}^{K}, \underline{t}^{K}\right\}_{K=1,2}\right\}$ represents the set of kinematic parameters to be determined; $\underline{X}_{j}^{I}$ stands for the $j$-th grid point in the $I$-th radial layer; $m$ and $n$ denote the numbers of layers and of correlation points per layer, respectively. $\|*\|$ is $\ell^{2}$ norm.

Finally, the strain tensor $\varepsilon$ is calculated from the five kinematic parameters:

$$
\boldsymbol{\varepsilon}=\left[\begin{array}{ccc}
u_{r, r_{0}} & \frac{1}{2} r_{0}\left(\lambda, r_{0} z_{0}+\beta_{z, r_{0}}\right) & \frac{1}{2}\left(\varepsilon_{z z, r_{0}} z_{0}+T_{z, r_{0}}\right) \\
\frac{1}{2} r_{0}\left(\lambda, r_{0} z_{0}+\beta_{z, r_{0}}\right) & \frac{u_{r}}{r_{0}} & \frac{1}{2} \lambda r_{0} \\
\frac{1}{2}\left(\varepsilon_{z z, r_{0}} z_{0}+T_{z, r_{0}}\right) & \frac{1}{2} \lambda r_{0} & \varepsilon_{z z}
\end{array}\right]
$$

The components are uniform for each layer, and those involving the radial derivative $\left(*, r_{0}\right)$ are evaluated by finite difference for two adjacent radial layers $(I$ and $I+1)$.

Thus evaluated average strains at each radial position combines the DVC measurements of the two separate volume sets taken from two different vertical 
positions. Their radial profiles provide an indicator of damage distribution throughout the tube thickness at a "semi-macroscopic" length scale.

\subsection{Crack detection}

A recently developed image subtraction technique [20,25] based on the DVC displacement field is used to detect cracks in the material. The image subtraction is performed within the reference configuration, where every image voxel at position $\underline{X}$ is initially associated with a reference gray level $f(\underline{X})$. A voxel-wise transformation field $\Phi$ between the reference and deformed images is continuously built throughout the whole image by interpolation of the coarse DVC measurements. Note that DVC measurements showing bad correlation values are excluded from the interpolation (e.g. the correlation subsets overlapping cracks). According to the voxel-wise transformation field, the reference voxel $\underline{X}$ is then given a deformed gray level $g(\Phi(\underline{X})$ ), which is deduced from the deformed image, making use of a tricubic gray level interpolation. The gray level function of the subtracted image $r(\underline{X})$ is obtained by

$$
r(\underline{X})=\frac{1}{2}\left[g(\Phi(\underline{X}))-f(\underline{X})+r_{m}\right]
$$

where $r_{m}$ is an offset ensuring the consistency of image format, e.g. $r_{m}=255$ for 8bit images. Details on this procedure can be found in [20] and in [24] (Chapter 2) for the parameters used for the present study.

Image subtraction is performed at each loading step, separately for the two sequential images, and the resulting subtracted images are stitched into a single one by simple juxtaposition [24]. Within the subtracted image the contrast due to the composite microstructure is expected to be removed, and the remaining contrast should be attributed only to strong local kinematic fluctuations (cracks) that are not taken into account by the mesoscale low-pass interpolated transformation $\Phi(\underline{X})$. However, in practice, image artifacts still remain (Fig.4.b), and they must be carefully dealt with to reduce their effects on further quantitative measurements. Specific procedures, whose details can be found in [24], are applied for this purpose. This artifact processing is overviewed in Fig.4.c-f. First, the radial fluctuation of brightness is reduced (Fig.4.C) by subtracting the background gray levels from the original image, with the background gray levels being calculated from the average along each circumference around the rotation axis. Thus, a unique gray-level threshold can be applied for binarization (Fig.4.d). Then, the over-contrasted solid-pore interfaces are located through a Sobel filter in the reference image and then deleted from the binary image (Fig.4.e). Finally, the ring artifacts are removed using a method similar to that proposed by [26], together with small objects of volume size below 20 voxels. The final result of crack detection is illustrated in Fig.4.f. 

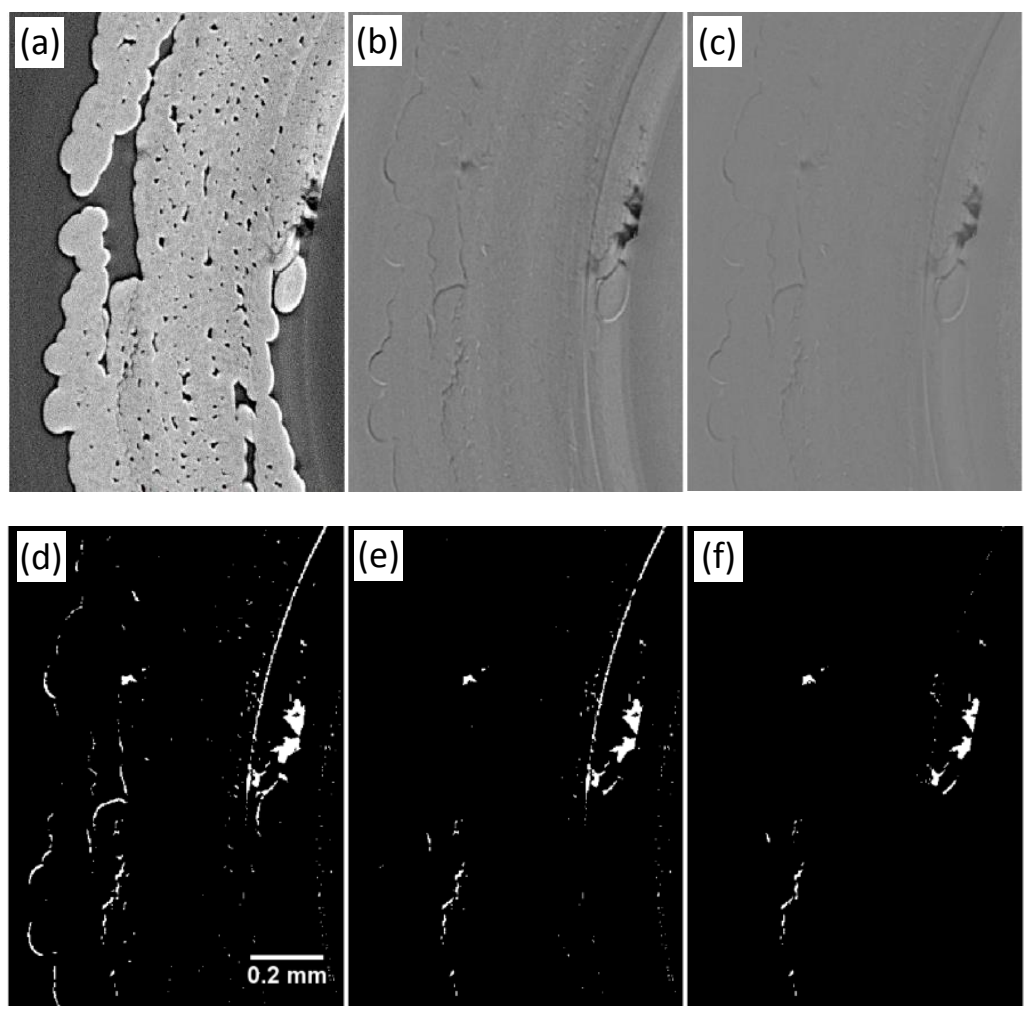

Fig.4 Overview of the procedures for crack detection: (a) deformed image, (b) subtracted image, (c) after reduction of radial brightness fluctuation, (d) after binarization, (e) after removal of over-contrasted solid-pore interfaces, (f) after ring removal.

\subsection{Crack quantification}

The crack detection procedure identifies the clusters of voxels that represent cracked volume. However, it is still not straightforward to deduce quantitative "surface" information, such as crack area, orientation or opening, from clusters of voxels. The purpose of the present section is to derive a complete methodology tackling this issue. First, it is introduced from a theoretical point of view in sections 3.3.1 and 3.3.2. Then, we use it to evaluate the local position, extension, orientation and opening of the detected cracks. Finally, the evaluated quantities are integrated over the region of interest to derive global and averaged quantities.

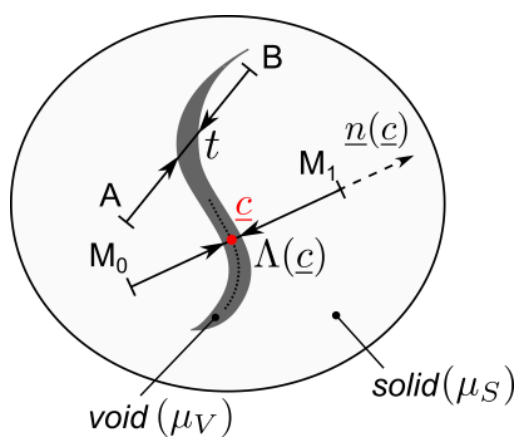

Fig. 5 X-ray paths through a crack in a homogeneous material. 


\subsubsection{Preamble: quantification of cracks in homogeneous medium}

To introduce the quantification methodology, we first consider a simpler case of a homogeneous material that contains a network of cracks. We consider a particular crack in this network and an arbitrary linear path going from point $A$ to point $B$ through the crack (see Fig.5). According to Beer-Lambert law, the attenuation of Xray along this path is given by

$$
-\ln \frac{N_{A}}{N_{B}}=\int_{A}^{B} \mu(X) \mathrm{d} \underline{X}
$$

where $N_{A}$ and $N_{B}$ quantify the fluxes of photons of an X-ray along the $\overrightarrow{A B}$ direction before and after crossing the segment $A B, \mu(\underline{X})$ is the attenuation coefficient at each position $\underline{X}$. We assume the attenuations of the solid matrix and the void phase (air) are uniform and given by coefficients $\mu_{S}$ and $\mu_{V}$, respectively. Thus, the attenuation along the path $A B$ can be written as:

$$
\int_{A}^{B} \mu(\underline{X}) \mathrm{d} \underline{X}=(\|\overrightarrow{A B}\|-t) \cdot \mu_{S}+t \cdot \mu_{V}
$$

where $t$ is the path length of the X-ray through the crack. This quantity can then be expressed as follows

$$
t=\int_{A}^{B}\left(\frac{\mu(\underline{X})-\mu_{S}}{\mu_{V}-\mu_{S}}\right) \mathrm{d} \underline{X}
$$

We assume hereafter that cracks are essentially $2 \mathrm{D}$ objects in 3D space, so they can be characterized by a mean surface with a local normal $\underline{n}(\underline{c})$ and local thickness $\Lambda(\underline{c})$ for any arbitrary position $\underline{c}$ on the mean surface (see Fig.5). Let us now consider an X-ray propagating through $\underline{c}$ and along $\underline{n}(\underline{c})$. Eq.8 provides the local thickness of the crack, i.e. $t=\Lambda(\underline{c})$. By definition, an ideal X-ray absorption tomographic image is a 3D map of attenuation coefficient $\mu(\underline{X})$, so that any integration of this type will directly provide the thickness of the crack, as soon as the local normal direction is known and the path $M_{0} M_{1}$ fully encompasses the crack. This integration requires no preliminary accurate segmentation of the crack domain, which ensures the efficiency of the proposed method.

In a real tomographic image, we do not have the access to the local physical attenuation coefficient, but only an approximation obtained from the tomographic device. We recall some features of digital XRCT images. First, an XRCT image is a discrete representation of the continuous attenuation field, which is characterized by a voxel size and limited by spatial resolution of the setup. Second, it is composed of gray levels, which are usually assumed as an affine function of attenuation coefficients. Third, gray levels are affected by image noise. As a result, the gray level $f\left(\underline{X}^{i}\right)$ available for each voxel $i$ located at position $\underline{X}^{i}$ in a tomographic image reads

$$
f\left(\underline{X}^{i}\right)=a \cdot \int_{V\left(\underline{X}^{i}\right)}\left[K\left(\underline{y}-\underline{X}^{i}\right) \cdot \mu(\underline{y})\right] \mathrm{d} \underline{y}+b+f_{i}^{\prime}
$$


where $a$ and $b$ are the parameters of the affine function between attenuation coefficients and gray levels, $f_{i}^{\prime}$ is a random noise at voxel $i$, and the kernel $K(\underline{y})$ characterizes the spatial resolution of the imaging system. By means of some interpolation procedure, this discrete description can be extended to a continuous one $f(\underline{X})$ for every position $\underline{X}$. By analogy to Eq.8, an estimate of $\Lambda(\underline{c})$ can be obtained from the continuous gray-level function $f(\underline{X})$ :

$$
\Lambda(\underline{c}) \approx \int_{M_{0}}^{M_{1}}\left(\frac{f(\underline{X})-f_{S}}{f_{V}-f_{S}}\right) d \underline{X}
$$

where $f_{S}$ and $f_{V}$ stand for the gray levels in solid and void, respectively. This estimate provides a weighted average of the crack thickness over a typical length linked to the image spatial resolution (see Appendix A). In practice, it can be obtained by a numerical integration along a sufficiently long path normal to the crack (longer than the spatial resolution of the image). This integration will be presented in section 3.3.4. Its subvoxel accuracy will be limited by the image noise with respect to the image contrast, i.e. $f_{i}^{\prime} /\left(f_{V}-f_{S}\right)$, and by the approximation related to the gray level interpolation [20]. More details about the relation between Eq.8 and 10 are given in Appendix A. It should be noted that in Eq.8, $\mu(\underline{X})$ equals either $\mu_{V}$ or $\mu_{S}$, whereas in Eq.10, $f(\underline{X})$ takes intermediate values between $f_{V}$ and $f_{S}$, because a real tomographic image provides a blurred vision of the real crack. The ratio $(f(\underline{X})-$ $\left.f_{S}\right) /\left(f_{V}-f_{S}\right)$ can be interpreted as a local void volume fraction at position $\underline{X}$ in the context of a diffuse description of cracks.

\subsubsection{Quantification from subtracted image}

The previous methodology enables us to quantify cracks in ideal homogeneous materials. Now we extend it to heterogeneous materials, in which cracks are detected from the comparison between reference and deformed images (see section 3.2).

Let now $f(\underline{X})$ describe the continuous gray level distribution in the reference image of the undamaged solid and $g(\underline{x})$ describe the gray levels in the corresponding volume in the deformed image, in which a crack has appeared and could be detected with the subtraction methodology [20]. As previously, the crack is essentially a flat structure and can be given a normal direction, perpendicular to its mean surface. Let $m_{0} m_{1}$ be a segment crossing the crack along its normal direction in the deformed configuration (see Fig.6). The crack thickness is again denoted as $\Lambda$, and it is delimited by $m_{i}$ and $m_{f}$. According to the mesoscopic mechanical transformation $\Phi$ evaluated by DVC, which is locally affine, the corresponding segment $M_{0} M_{1}$ and limits $M_{i}$ and $M_{f}$ can be defined in the reference configuration. The virtual crack, obtained by transforming the real one in the deformed configuration back to the reference image, is drawn in dashed line in Fig.6. 


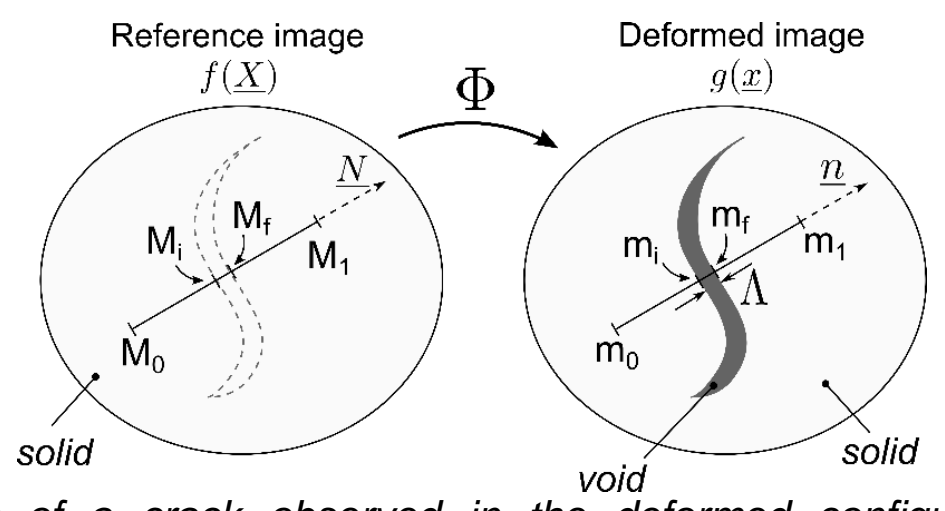

Fig.6 Schematic of a crack observed in the deformed configuration, which is transformed back to the undamaged reference configuration (dashed lines)

For the sake of clarity, we consider the subtracted image in its simplest form:

$$
r_{0}(\underline{X})=g(\Phi(\underline{X}))-f(\underline{X})
$$

Apart from image noise, $r_{0}$ is observed to be zero outside the detected crack. This would be exactly true if the transformation $\Phi$ is the true mechanical transformation, because of the classical assumption of conservation of gray level on which DVC relies. It is also true in the case when $\Phi$ is the mesoscopic approximation of the actual transformation based on a limited local image contrast. Indeed, in this case two situations are encountered in practice. Either there is some physical contrast in the images, provided for instance by micropores, and DVC makes use of it to locally map properly the reference and deformed images; or such contrast is not available, as is the zone made of pure dense $\mathrm{SiC}$, and both images exhibit the same locally uniform gray level, so that the image difference will be zero even if the mapping is incorrect. As a consequence, the integral along $M_{0} M_{1}$ can be written as

$$
\int_{M_{0}}^{M_{1}} r_{0}(\underline{X}) \mathrm{d} \underline{X}=\int_{M_{i}}^{M_{f}}[g(\Phi(\underline{X}))-f(\underline{X})] \mathrm{d} \underline{X}
$$

Consider now first that tomographic images are perfect, i.e. with infinitely small voxels or infinitely high spatial resolution. Then the gray levels of crack in the deformed image coincide with those of air and they are uniform, equal to $f_{V}$. We need to assume that the gray levels in the solid phase located along $\overrightarrow{M_{l} M_{f}}$ (that will be replaced by void after cracking) in the reference image are also uniform along this little segment, equal to $f(\underline{C})$ with $\underline{C}$ denoting a point at the mean surface of the crack transformed back to the reference configuration. Then, the integral becomes

$$
\int_{M_{0}}^{M_{1}} r_{0}(\underline{X}) \mathrm{d} \underline{X}=\left(f_{V}-f(\underline{C})\right)\left\|\overrightarrow{M_{l} M_{f}}\right\|
$$

Let $\epsilon_{\text {mes }}$ stands for the evaluated mesoscopic strain along $M_{i} M_{f}$, identical to the one along $M_{0} M_{1}$, then we have.

$$
\left\|\overrightarrow{m_{l} m_{f}}\right\|=\Lambda=\left(1+\epsilon_{m e s}\right)\left\|\overrightarrow{M_{l} M_{f}}\right\|
$$


Combining Eq.13, and using the relations $f(\underline{X})=f(\underline{C})$ for $\underline{X} \in \overrightarrow{M_{l} M_{f}}$ and $r_{0}(\underline{X})=0$ for $\underline{X} \in\left\{\overrightarrow{M_{0} M_{l}} \cup \overrightarrow{M_{f} M_{1}}\right\}$, the crack opening is given by

$$
\Lambda=\left(1+\epsilon_{m e s}\right) \int_{M_{0}}^{M_{1}} d_{0}(\underline{X}) \mathrm{d} \underline{X} \text {, with } d_{0}(\underline{X})=\frac{g(\Phi(\underline{X}))-f(\underline{X})}{f_{V}-f(\underline{X})}
$$

As will be discussed later in Section 3.4, the mesoscopic deformation $\epsilon_{\text {mes }}$ in such ceramic material is usually small enough to be negligible. Then the crack opening can be estimated as $\Lambda \cong \int_{M_{0}}^{M_{1}} d_{0}(\underline{X}) \mathrm{d} \underline{X}$.

Consider now the real tomographic images, which are blurred versions of the ideal ones (Eq.9). We can use the similar reasoning as in the case of a homogeneous material (Appendix A). Under the assumption that the segment $M_{0} M_{1}$ is sufficiently large with respect to image resolution, Eq.15 provides a measurement of crack thickness $\Lambda$ that is locally averaged according to image resolution, as explained in Appendix A. It should be noted that the coefficient $\left(f_{V}-f(\underline{C})\right)$ on the right-hand side of Eq.13 is not influenced by blurring, because of the uniformity of the gray level in air and the assumed local uniformity of the matrix around point $\underline{C}$.

Local crack thickness can thus be evaluated by integrals of the continuously interpolated subtracted experimental images along sufficiently long segments normal to the crack mean surface. Accuracy of this evaluation is essentially controlled by the ratio of the noise in subtracted images (which is equal to $\sqrt{2}$ times the noise in a single image) to the local matrix-void contrast $\left(f_{V}-f(\underline{C})\right)$. The practical implementation of this methodology requires first a voxel-wise computation of the local damage $d_{0}(\underline{X})$, then an evaluation of the local normal directions of the cracks detected in section 3.2, finally the through-thickness integration of the continuously interpolated damage $d_{0}$. Details of these procedures are provided in the next sections.

\subsubsection{Voxel-wise damage description}

Considering the practical definition of the subtracted image used to detect crack and defined in Eq.5, the reasoning exposed in section 3.3.2 applies and leads to the following expression of the local crack opening:

$$
\Lambda \cong \int_{M_{0}}^{M_{1}} d(\underline{X}) d \underline{X}, \text { with } d(\underline{X})=\frac{2 \cdot r(\underline{X})-r_{m}}{f_{V}-f(\underline{X})}
$$

where $d(\underline{X})$ is defined as a voxel-wise damage variable, which should be theoretically bounded by 0 and 1 , with the two bounds being reached for non-cracked and fully cracked voxels, respectively. In practice, it is calculated for the crack voxels $\underline{X}_{c}$ resulting from the detection procedure only, leading to:

$$
d(\underline{X})=\left\{\begin{array}{cc}
\left\langle\frac{2 \cdot r(\underline{X})-r_{m}}{f_{V}-f(\underline{X})}\right\rangle & \text { if } \underline{X}=\underline{X}_{c} \\
0 & \text { otherwise }
\end{array}\right.
$$


where the symbol $\langle\cdot\rangle$ stands for truncating the values out of the range $[0,1]$, so that $d \in[0,1]$. In fact, image noises and artifacts lead to out-of-range values in a very limited number of voxels. The practical choice of the gray level $f_{V}$ in void will be discussed in section 3.4 .

In addition to this scalar quantity $d(\underline{X})$, for every crack voxel we define an orientation providing the local normal direction to the crack surface. It is calculated from the inertia tensor of a neighboring box centered at each considered crack voxel. The damage field $d(\underline{X})$ is used to weight the voxels in the neighboring box in this calculation. The eigenvector associated with the largest eigenvalue of this inertia tensor is defined as the normal vector $\underline{N}\left(\underline{X}_{c}\right)$ of the crack voxel. It provides an orientation angle $\gamma_{r}\left(\underline{X}_{c}\right)$ between the normal vector $\underline{N}\left(\underline{X}_{c}\right)$ and the radial axis $\underline{e}_{r}\left(\underline{X}_{c}\right)$ of the local coordinates (Fig.7.a). The size of the neighboring box is an important parameter to be optimized. It must be large enough to weaken the effect of discretization and noise, and small enough to provide sufficiently local characteristics. Accordingly, after some trials on the largest detected cracks, it is set to $11 \times 11 \times 11$ voxels in the present analysis.

According to the orientation angle $\gamma_{r}$ (Fig.7.b), we can define two sets of crack voxels: those with an angle larger than $\pi / 4$ belong to circumferential cracks, and the others to in-plane cracks. Although the term "in-plane" is not rigorously correct for a tubular composite, we use it to describe the cracks that grow along the tube wall.

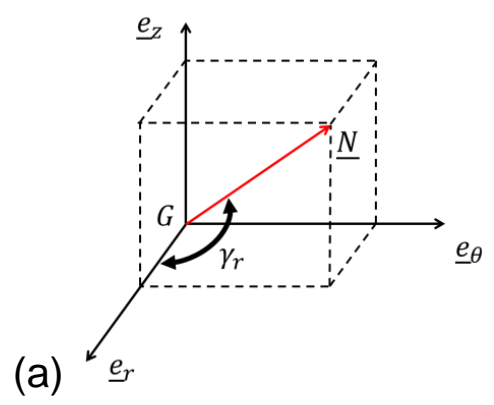

(b)

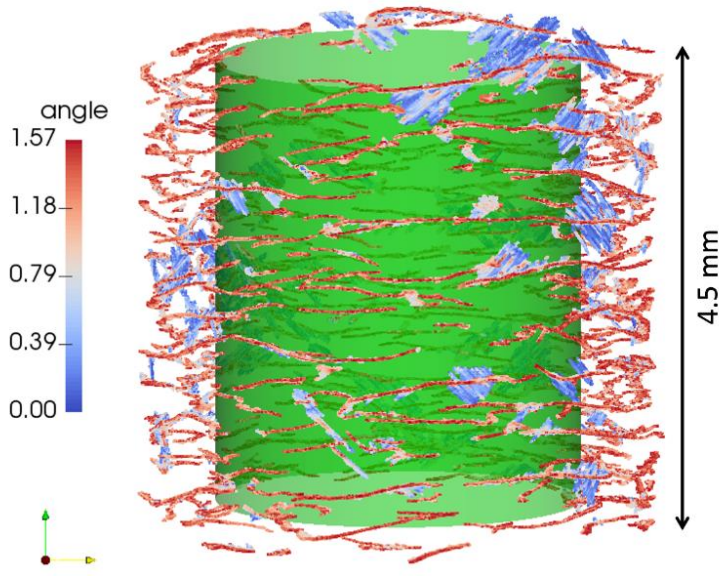

Fig.7 (a) Illustration of the characteristic angle $\gamma_{r}$ for crack orientation. (b) The 500 largest detected cracks colored according to their orientation angle $\gamma_{r}\left(\underline{X}_{c}\right)$, the green cylinder represents the internal free surface of the tube. 


\subsubsection{Local crack opening and orientation}

Now we propose a procedure for assembling the voxel-wise information into surface information, as illustrated in Fig.8. The first step consists in computing the integral of the discrete damage field through the crack thickness for every crack voxel and projecting it onto a mean crack surface. To do so, for each crack voxel $\underline{X}_{c}$, we construct a discrete damage profile $d\left(\underline{X}_{c}^{h}\right)$ along the normal vector $\underline{N}\left(\underline{X}_{c}\right)$. The discretization points $\underline{X}_{c}^{h}$ are defined by a 1-D discrete coordinate $h$ with respect to the related normal vector:

$$
\underline{X}_{c}^{h}=\underline{X}_{c}+h \cdot \underline{N}\left(\underline{X}_{c}\right)
$$

In practice, $h$ is defined within the interval $[-3,3]$ (voxels) with an increment of one voxel, this interval being thicker than all detected cracks, and larger than image spatial resolution as required from the discussion in section 3.4. It should be noted that the profile value $d\left(\underline{X}_{c}^{h}\right)$ has been determined from a trilinear interpolation of the damage field $\underline{d(\underline{X})}$. The projected position $\underline{X}_{c}^{p}$ of the crack voxel is then defined as the barycenter position $\underline{X}_{c}\left(h^{m}\right)$ of the damage profile $d\left(\underline{X}_{c}^{h}(h)\right)$ :

$$
\underline{X}_{c}^{p}=\underline{X}_{c}+h^{m} \cdot \underline{N}\left(\underline{X}_{c}\right) \quad \text { with } h^{m}=\frac{\int_{-3}^{3}\left[d\left(\underline{X}_{c}^{h}(h)\right) \cdot h\right] d h}{\int_{-3}^{3} d\left(\underline{X}_{c}^{h}(h)\right) d h}
$$

Consistently with Eq.15, the local opening $\Lambda$ is then evaluated for each projected position from the integration of the damage profile:

$$
\Lambda\left(\underline{X}_{c}^{p}\right)=\int_{-3}^{3} d\left(\underline{X}_{c}^{h}(h)\right) d h
$$

This operation is performed for all crack voxels, resulting in a large set of projected positions, each of them associated with a local opening $\Lambda\left(\underline{X}_{c}^{p}\right)$ and local normal vector $\underline{N}\left(\underline{X}_{c}^{p}\right)=\underline{N}\left(\underline{X}_{c}\right)$. Let us emphasize that the above integrals are not simple summations over voxel values, but true continuous integrations based on interpolated gray levels as required by Eq. 15 .

As illustrated in Fig.8.a, the second step consists in merging these spatially dispersed projected positions into a regular grid, in order to prepare for further surface integration. The initial image grid is used: the projected positions belonging to a same voxel are merged by averaging their locations, openings and normal vectors, leading to elementary projected position $\widetilde{X_{c}^{p}}$, elementary opening $\widetilde{\Lambda_{c}^{p}}$ and elementary normal vector $\widetilde{N_{c}^{p}}$, respectively. The result is illustrated in Fig.8.b. It should be noted that these quantities are scalar or vectorial ones, not rounded to integer values. 

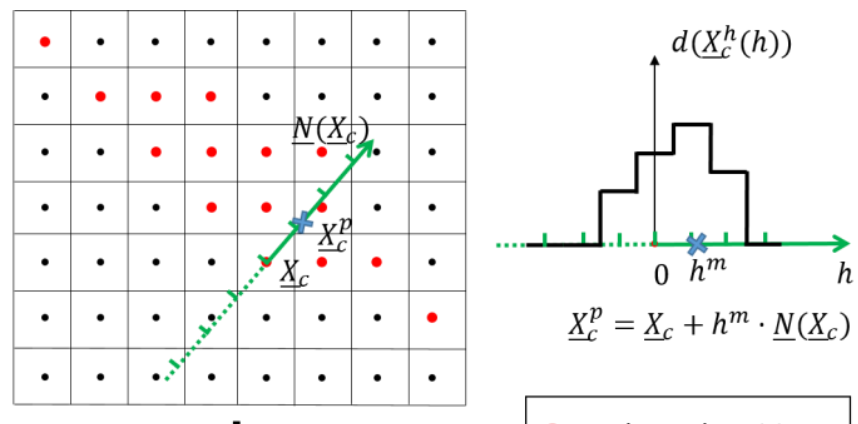

$\underline{X}_{c}^{p}=\underline{X}_{c}+h^{m} \cdot \underline{N}\left(\underline{X}_{c}\right)$

Crack voxel position

X Projected position
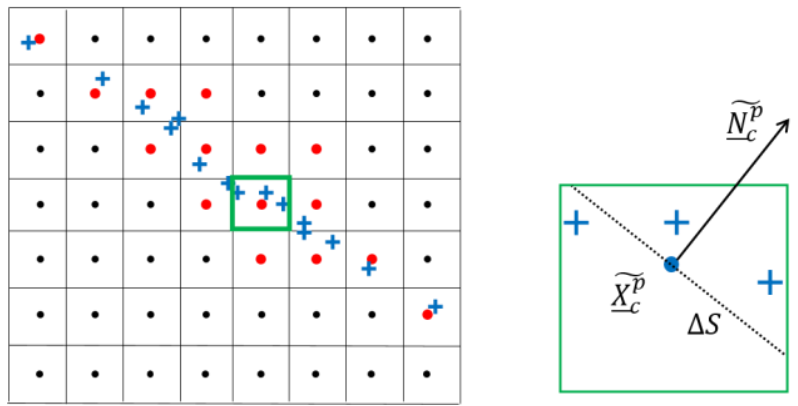

(a)

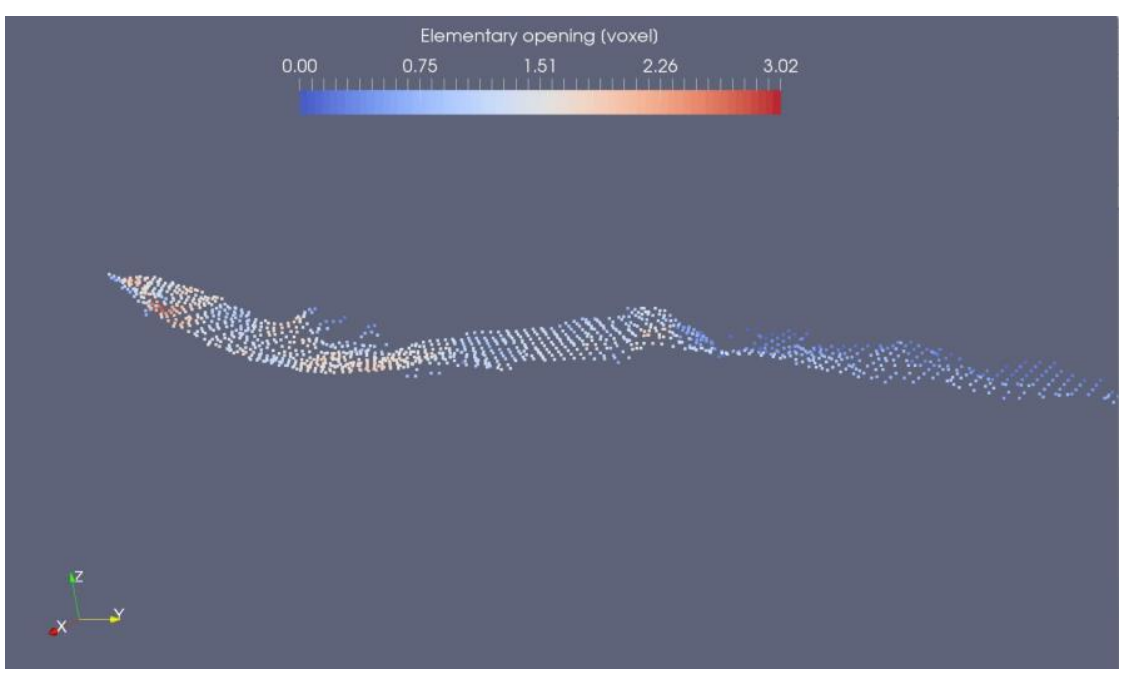

(b)

Fig.8 (a) Illustration of crack quantification procedure. (b) Local elementary openings at the projected positions for a selected crack (video visualization can be found in the supplementary files). 


\subsubsection{Crack surface area and average opening}

The accuracy of the quantification result relies on the statistics of the measurements. Due to the error sources, as will be addressed in section 3.4, the proposed method is recommended to be used for large crack colonies instead of individual small cracks. Therefore, we propose to calculate total crack surface area and average crack opening as follows.

For each voxel with an elementary projected position $\widetilde{X_{c}^{p}}$ inside, an elementary crack area $\Delta S$ can be approximated as the intersection between the voxel and the plane defined by $\widetilde{\underline{X}_{c}^{p}}$ and the elementary normal vector $\widetilde{N_{c}^{p}}$. Thus, it is possible to integrate these elementary quantities over an appropriate set of crack voxels, so that the total surface area $S$ and average opening $\bar{\Lambda}$ are evaluated:

$$
\left\{\begin{array}{l}
S=\sum \Delta S \\
\bar{\Lambda}=\frac{\sum \widetilde{\Lambda_{c}^{p}} \cdot \Delta S}{S}
\end{array}\right.
$$

This set of voxels can be either all voxels in the sample or a set of connected crack voxels defining an individual crack, leading to "per-sample" or "per-crack" quantities, respectively. To further quantify the crack evolution at the scale of the sample, we define the crack surface density as $\rho=S / V_{S}$, where $V_{S}$ is the volume of the solid phase (non-pore phase) evaluated from a segmentation of the reference image.

\subsection{Discussions on error sources and applicability}

In this section, we address some sources of errors for the quantification procedure, and discuss its applicability to different situations.

First, even though the crack detection procedure is able to identify the cracks with subvoxel opening $[20,25]$, it still faces a detection threshold: cracks are not detectable below a minimum opening, which is of course first linked to the voxel size and then to the signal to noise ratios according to the statistical consideration developed in Appendix A. Errors linked to gray level interpolations might also be considered but are not addressed here for brevity. We refer to references $[20,27]$ for these discussions. Altogether, both the detection threshold and the error in thickness quantification have been be evaluated to be about $0.1 \sim 0.2$ voxel $(0.3 \sim 0.5 \mu \mathrm{m})$ in the present study. Such a limitation is inherent to any image-based characterization method. It is therefore crucial to restrict the interpretation of the results to a limited range of length scale, or conversely, to choose the appropriate image resolution (voxel size) according to the length scale to be investigated. In particular, the detection threshold in the present study leaves the very slightly opening cracks out of the analysis, especially those at fiber-matrix interfaces. As a result, we state that the further analysis is valid only at mesoscopic length scale of the braided $\mathrm{SiC} / \mathrm{SiC}$ composite tube. A more thorough characterization would require additional tomographic observations at smaller scales, at which the proposed image processing methods would still be applicable. 
Secondly, the phase-contrast edge enhancement effect could invalidate the assumption about the linear relationship between gray-level and crack opening. However, we have chosen the value of $f_{V}$ according to the gray levels of the micropores that are also affected by the phase contrast. This choice could compensate the related interference to some extent, but a reliable improvement would be to establish a more precise relationship by taking into account the phasecontrast effect more quantitatively. This is probably the major limitation of the current practical application of the presented general methodology.

Thirdly, we have neglected the mesoscopic strain $\epsilon_{\text {mes }}$ in Eq.15, which should be reasonable for quasi-brittle composite materials, yet cautions must be paid if the material under investigation is ductile or contains very fine contrasts in tomographic images. This is because cracks are identified within reference configuration from image subtraction. Non-zero gray values in subtracted image highlight the discontinuity (cracks) that has not been captured by DVC. We emphasis that the "poorly-corrected" correlation points are eliminated from the calculation of image subtraction, if the correlation criterion has been properly chosen. In fact, every voxel is associated with a transformation that is evaluated from interpolation over the neighboring "well-correlated" correlation points. This interpolated transformation is actually a measurement at the mesoscopic length scale, at which image contrast is enough for DVC calculation. In other words, the mesoscopic length scale used by image subtraction is linked to the correlation window size, which is $30 \sim 50$ voxels in the present study. The typical maximum crack opening of the studied composite is a few micrometers, i.e. 1 2 voxels. According to the image subtraction procedure, the mesoscopic strain $\epsilon_{\text {mes }}$ in Eq.15 can be roughly estimated as $\epsilon_{\text {mes }} \approx \lambda /(2 D)$, with $\lambda$ the actual crack opening and $D$ the correlation window size. Therefore, $\epsilon_{\text {mes }}$ is much less than one, and hence negligible. However, the influence of $\epsilon_{\text {mes }}$ may become significant, when the crack opening is quite large (i.e. in ductile materials), or/and the correlation window is small (i.e. the microstructure provides fine image contrast). In the case of well-textured image with enough local contrasts for DVC, it would be more relevant to make direct use of DVC-measured displacement field to detect and quantify the cracks, as proposed by [28]. In fact, our method offers an alternative solution to crack detection and quantification in the case that image contrast is not local enough, which is commonly encountered for CMCs.

Furthermore, as a common feature of many field-based measurements such as imaging and full-field strain measurements, the proposed quantification method provides a better accuracy for the evaluation of the average opening of large populations of cracks than the local opening of a small crack, because of statistical averaging of random noises and artifacts associated with independent measurements. This, however, does not prevent the application of the proposed method to a single crack, though caution must be paid regarding the reduced measurement accuracy.

Concerning the effect of possible artifacts induced by image post-processing, we emphasis on the fact that the subtracted image has been directly used for the quantification of crack opening without any filtering. Filters have only been used to select the crack voxels in the segmented subtracted image to separate them from artifacts, so they have no effect on the subtracted image nor on the integration along the normal directions. Also, the sensitivity of the reconstruction parameters should have been reduced by the fact that $f_{V}$ and $f_{S}$ have been adapted to the actual 
reconstructed image. Therefore, measurement biases related to image processing (including reconstruction) are believed to be minor compared to the other error sources outlined above.

Let us finally mention that the term "crack opening" in this paper should be distinguished from the well-known crack opening displacement (COD), because the proposed method cannot properly decompose the three components of the discontinuity on the two sides of a crack. Indeed, the present gray-level based method provides an estimate of the normal component of a COD only, with the normal direction determined from the 3D geometry of the considered crack.

\section{Results and discussion}

Some results are selected in this section to demonstrate the capability of the proposed methods. The in-depth interpretations of the results on the material itself will be presented in a separate paper together with two other samples with different braiding angles [29].

\subsection{Radial profiles of semi-macroscopic strains}

The overall strains are measured for each radial layer according to section 3.1. To validate the measurement method, the DVC-measured strains at the outer layer of the tube are compared to those continuously measured with extensometers during two macroscopic tensile tests (Fig.9.a). The observed differences within these reference curves for the hoop strain $\left(\varepsilon_{\theta \theta}\right)$ could be attributed to the limited precision of the transverse extensometer positioned on the rough surface of a small tube (diameter $\sim 5 \mathrm{~mm}$ ). Nevertheless, the DVC-measured strains are still in good agreement with the extensometry measurements, especially for the axial strain $\left(\varepsilon_{z z}\right)$. This indicates that (i) the measurement method, involving DVC and transformation fitting, provides a satisfactory accuracy, and (ii) the macroscopic behavior of the studied material is highly reproducible even under different loading systems.

The four average strain components are presented in Fig.9.b at various radial positions for all five loading levels. The overall magnitudes of the three strains $\left(\varepsilon_{r r}\right.$, $\left.\varepsilon_{\theta \theta}, \varepsilon_{z z}\right)$ increase monotonically with the tensile load. The axial strain is uniform throughout the tube thickness. Surprisingly, the radial strain profile exhibits positive, and non-uniform, values over the thickness, indicating a negative Poisson's ratio. Furthermore, the absolute value of hoop strain decreases from the inner side to the outer side of the tube. In fact, these strain distributions are related to the opening of the in-plane cracks. These results will be discussed in detail in a separate paper [29] for conciseness in the present paper. 


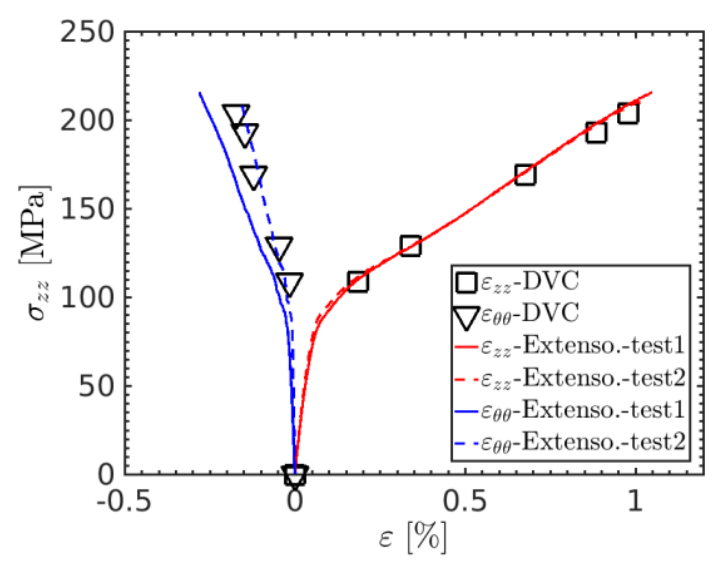

(a)

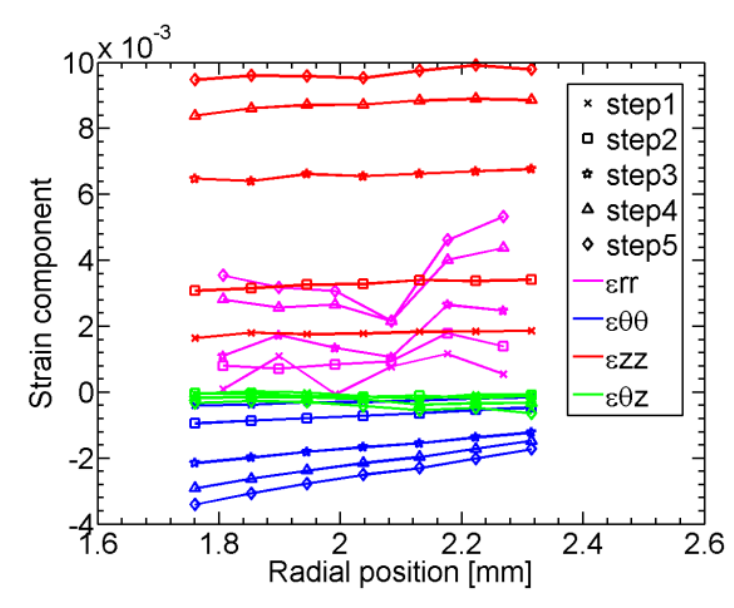

(b)

Fig.9 (a) Comparison between the strains measured on the outer layer by DVC and those measured with standard extensometer. (b) Radial profiles of overall strains measured by the transformation fitting method.

\subsection{Damage characterization}

In the radial direction, the microstructure is heterogeneous due to the braid architecture and can be indicated by the distribution of macropores (dashed line in Fig.10). The three highest peaks reveal the locations of the inner surface $(\sim 1.8 \mathrm{~mm})$, the outer surface $(\sim 2.45 \mathrm{~mm})$ and the braided layer interface $(\sim 2.15 \mathrm{~mm})$, respectively. The two lower peaks correspond to the mid-parts of each braided layer where tows cross each other.

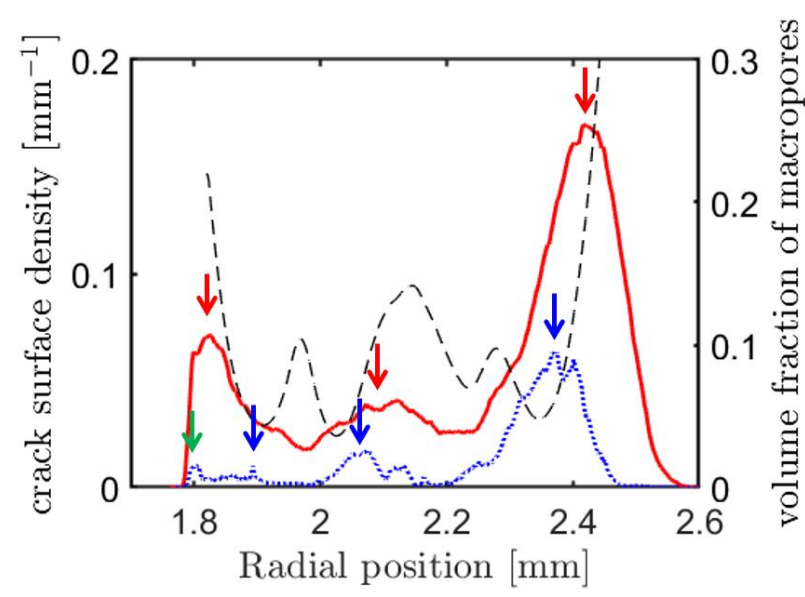

Fig.10 Radial profiles of surface density of circumferential cracks (red) and in-plane cracks (blue) through the tube thickness at the last loading step. The profile of pore volume fraction is shown (dashed line) to indicate the woven fiber architecture throughout the tube thickness. Arrows indicate the crack populations that are discussed in the main text. 
The through-thickness profiles of both circumferential and in-plane cracks (see section 3.3.3 for definition) are superimposed in Fig.10. Three populations (the three primary peaks in red in Fig.10) of circumferential cracks appear at the inner and outer free surfaces of the tube and at the boundary between two braided layers. The locations of these primary peaks are consistent with those of the macropores, which indicates that the detected circumferential cracks appear mostly in the zones where most peripheral matrix has been deposited during the CVI process. As for in-plane cracks (Fig.10.b), four primary peaks are highlighted and their positions are in agreement with the zones with few macropores, except the internal one $(\sim 1.8 \mathrm{~mm})$, which could be linked to the matrix debonding from the polished tube inner surface, as confirmed in Fig.11 (pointed out by the dotted arrow). The cross-section in Fig.11 suggests that the in-plane cracks seem to propagate through the micropores inside the tows but not along the tow boundaries. This observation is also in agreement with the relative positions of the peaks in Fig.10. It is useful to mention that the crack surface density at each radial position of the profile in Fig.10 has been evaluated within a thin circumferential cylinder with a thickness of 2 voxels, so there are about 200 300 independent measurements through the tube thickness.

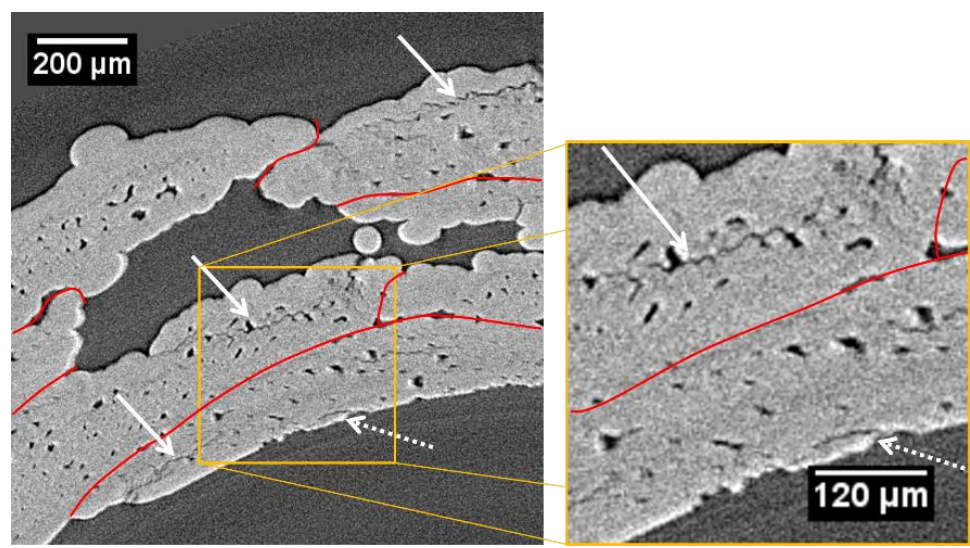

Fig.11 Cross-sectional slice showing in-plane cracks appearing at different radial positions. Tow boundaries are marked by red lines. The dotted arrow indicates an example of matrix debonding.

In the same manner, the through-thickness profile of the average crack opening of circumferential cracks is evaluated and plotted in Fig.12. The locations of larger openings (three peaks) are consistent with the three crack-rich zones. Overall, the outer surface of the tube exhibits larger crack opening. It is worth mentioning that the average opening measured at the outer surface by the current method $(\sim 2.5 \mu \mathrm{m})$ is very similar as the measurement in [19] (Fig.5 in the reference), where precise DIC measurement was used to quantify the displacement jumps at the surface cracks of a $\mathrm{SiC} / \mathrm{SiC}$ tube. 


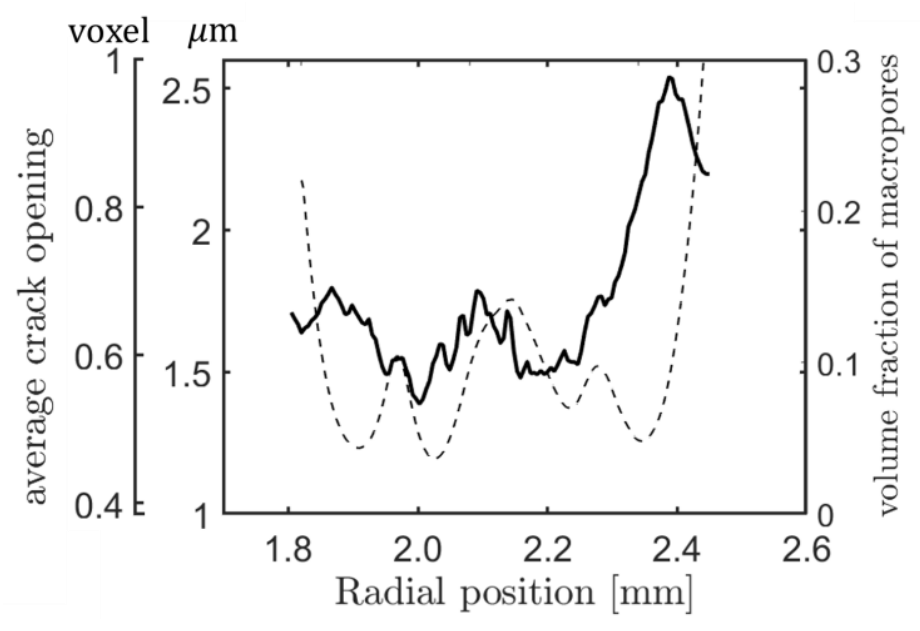

Fig.12 Radial profile (solid line) of the average opening of circumferential cracks detected at the last loading step. The profile of pore volume fraction is shown (dashed line) to indicate the woven fiber architecture throughout the tube thickness.

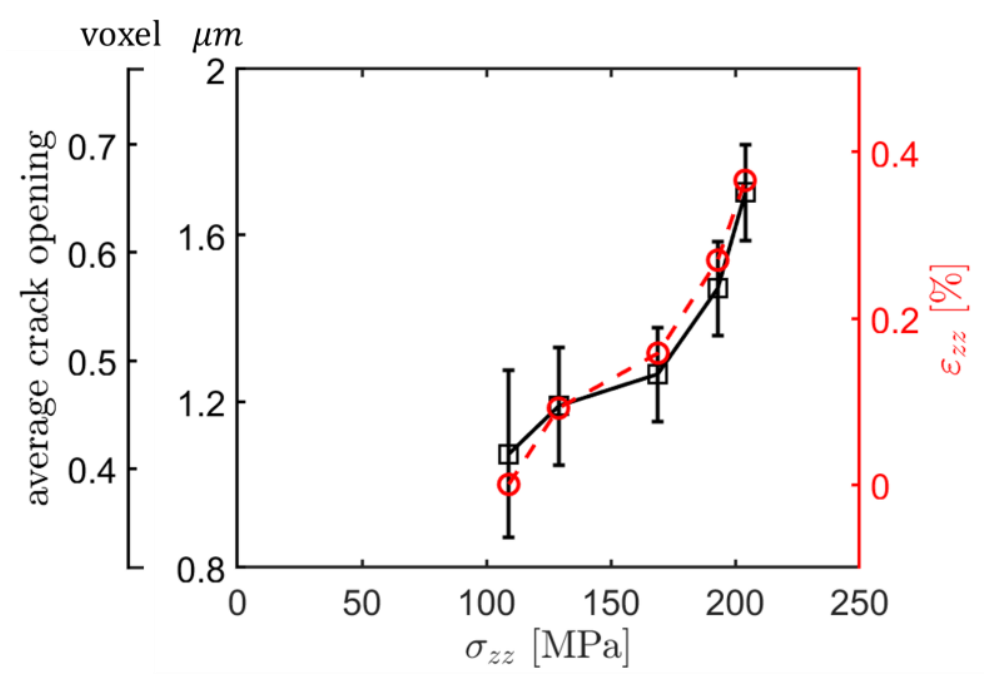

Fig. 13 Evolution of average opening $\bar{\Lambda}$ of in-plane cracks (solid line), compared with that of the average radial strain $\varepsilon_{r r}$ (red dashed line).

Finally, as an attempt to correlate the detected damage to the macroscopic behavior, the evolution of the average opening of the detected in-plane cracks is compared to the average radial strain $\varepsilon_{r r}$ (Fig.13). The strain value is computed by averaging the measures at all radial positions from Fig.9.b. The evolution of the average opening of in-plane cracks and the average radial strain are nicely consistent. This suggests that the unusual positive radial strain could be mainly due to the opening of the in-plane cracks. 


\section{Conclusions}

In this paper, a series of methodologies are introduced, developed and applied to real tomographic images, which could thus be fully analyzed to detect and characterize damage in a braided composite tube. A semi-macroscopic method is used to measure the heterogeneous deformation through the tube thickness, and validated against surface extensometry measurement. After the DVC-based image subtraction and a careful processing of image artifacts, cracks with complex geometries are detected within the microstructure. A quantification method based on the physical meaning of the gray levels in absorption contrast tomography images is proposed to measure two geometric parameters of the detected cracks: opening and surface area. Although used here on the cracks detected from the subtraction of sequential in situ images, the proposed method is generic and is also applicable to objects that can be segmented from one single image of a homogeneous material, as discussed in section 3.3.1. Whilst improvements are still expectable for this method, it provides a new idea in characterizing the local and global geometries of $3 \mathrm{D}$ crack networks in CMCs using XRCT, which is more relevant than standard voxel counting methods. Some selected results are presented to illustrate the capability of the techniques proposed in this paper. The crack quantification method will be validated in the future on other materials with only a few large cracks inside.

\section{Acknowledgements}

This work was supported by the CNRS program "Défi NEEDS Matériaux". The loading machine used during the in situ XRCT test was designed and manufactured by LMS (Ecole polytechnique) and Laboratoire Navier.

\section{Appendix A}

We provide here a detailed demonstration that links up the definition of crack thickness in Eq.8 and its diffuse evaluation in Eq.10. Substituting $f(\underline{X}), f_{S}$ and $f_{V}$ by the definition of Eq.9, the right-hand side of Eq.10 becomes

$$
\begin{aligned}
\int_{M_{0}}^{M_{1}}\left(\frac{f(\underline{X})-f_{S}}{f_{V}-f_{S}}\right) \mathrm{d} \underline{X} \\
\quad=\int_{M_{0}}^{M_{1}} \int_{V(\underline{X})} K(\underline{y}-\underline{X}) \frac{\mu(\underline{y})-\mu_{S}}{\mu_{V}-\mu_{S}} \mathrm{~d} \underline{y} \mathrm{~d} \underline{X}+\int_{M_{0}}^{M_{1}}\left(\frac{f^{\prime}(\underline{X})}{f_{V}-f_{S}}\right) \mathrm{d} \underline{X}
\end{aligned}
$$

Using the change of variable $\underline{H}=\underline{y}-\underline{X}$ and applying Fubuni's theorem, one obtains:

$$
\begin{aligned}
\int_{M_{0}}^{M_{1}}\left(\frac{f(\underline{X})-f_{S}}{f_{V}-f_{S}}\right) \mathrm{d} \underline{X}-\int_{M_{0}}^{M_{1}}\left(\frac{f^{\prime}(\underline{X})}{f_{V}-f_{S}}\right) \mathrm{d} \underline{X} \\
=\int_{M_{0}}^{M_{1}} \int_{V(\underline{o})} K(\underline{H}) \frac{\mu(\underline{X}+\underline{H})-\mu_{S}}{\mu_{V}-\mu_{S}} \mathrm{~d} \underline{H} \mathrm{~d} \underline{X}
\end{aligned}
$$




$$
\begin{aligned}
& =\int_{V(\underline{o})} K(\underline{H}) \int_{M_{0}}^{M_{1}} \frac{\mu(\underline{X}+\underline{H})-\mu_{S}}{\mu_{V}-\mu_{S}} \mathrm{~d} \underline{X} \mathrm{~d} \underline{H} \\
& =\int_{V(\underline{O})} K(\underline{H}) \cdot \Lambda(\underline{c}+\underline{H}) \mathrm{d} \underline{H}
\end{aligned}
$$

where $\Lambda(\underline{c}+\underline{H})$ is the physical crack thickness quantified through an integration along a path normal to the crack mean surface and passing through the point $\underline{c}+\underline{H}$ (see the definition of Eq.8). Let us decompose the vector $\underline{H}$ along its components parallel and normal to the crack mean surface, $\underline{H}=\underline{H}^{\|}+\underline{H}^{\perp}$. It is clear that $\Lambda(\underline{c}+\underline{H})=$ $\Lambda\left(\underline{c}+\underline{H}^{\|}\right)$, as long as the segment $M_{0} M_{1}$, translated by the vector $\underline{H}^{\perp}$, is long enough to still fully encompass the crack. One finally obtains

$$
\int_{M_{0}}^{M_{1}}\left(\frac{f(\underline{X})-f_{S}}{f_{V}-f_{S}}\right) \mathrm{d} \underline{X}=\Lambda^{K}(\underline{c})+\int_{M_{0}}^{M_{1}}\left(\frac{f^{\prime}(\underline{X})}{f_{V}-f_{S}}\right) \mathrm{d} \underline{X}
$$

where $\Lambda^{K}(\underline{c})=\int_{V(\underline{O})} K(\underline{H}) \cdot \Lambda\left(\underline{c}+\underline{H}^{\|}\right) \mathrm{d} \underline{H}$ is a "smoothed" crack thickness. This quantity is calculated as a weighted average of the local thickness over the width determined by the spatial resolution of the tomography device, which is usually of the order of a few voxels. As long as the latter is small with respect to the typical curvature radius of the real crack and to the typical length of the spatial variations of its thickness, the approximation $\Lambda(\underline{c}) \approx \Lambda^{K}(\underline{c})$ holds.

We emphasize that this result does not require the crack thickness to be large with respect to the image spatial resolution. Indeed, the accuracy of the evaluation of $\Lambda(\underline{c})$ can even be significantly better than voxel size and image resolution, and it is essentially governed by the last term in Eq.A.3, which characterizes the signal-tonoise ratio of the image. Assuming $f^{\prime}(\underline{X})$ to be a white noise at voxel scale, with zero statistical expectation, standard deviation $\sigma^{f}$, and correlation length equal to one voxel, it can be easily shown that $\int_{M_{0}}^{M_{1}}\left(\frac{f(\underline{X})-f_{S}}{f_{V^{-}}-f_{S}}\right) \mathrm{d} \underline{X}$ is an unbiased evaluation of $\Lambda^{K}(\underline{c})$ and that its standard deviation is given by

$$
\sigma^{\Lambda^{K}}=\frac{\sigma^{f} \sqrt{\left\|M_{0} M_{1}\right\|}}{\left|f_{V}-f_{S}\right|}
$$

This relation suggests that for optimal results, the integration segment should not be taken too large, so that $\sqrt{\left\|M_{0} M_{1}\right\|}$ remains close to unity. The best option is to take it as close as possible to the diameter of the support of the kernel $K(\underline{y})$. Rigorously speaking, the herein presented analysis ignores the periodic spatial dependence of the kernel $K$, which is induced by gray level interpolation. A more detailed, rather technical, analysis would be possible, but without significant change on the result, hence it is not presented here for conciseness. 


\section{References}

[1] L.L. Snead, T. Nozawa, M. Ferraris, Y. Katoh, R. Shinavski, M. Sawan, Silicon carbide composites as fusion power reactor structural materials, J. Nucl. Mater. 417 (2011) 330-339. doi:10.1016/j.jnucmat.2011.03.005.

[2] K. Yueh, K.A. Terrani, Silicon carbide composite for light water reactor fuel assembly applications, J. Nucl. Mater. 448 (2014) 380-388.

doi:10.1016/j.jnucmat.2013.12.004.

[3] J. Lamon, A micromechanics-based approach to the mechanical behavior of brittle-matrix composites, Compos. Sci. Technol. 61 (2001) 2259-2272. doi:10.1016/S0266-3538(01)00120-8.

[4] F. Bernachy-Barbe, L. Gélébart, M. Bornert, J. Crépin, C. Sauder, Anisotropic damage behavior of $\mathrm{SiC} / \mathrm{SiC}$ composite tubes: Multiaxial testing and damage characterization, Compos. Part A Appl. Sci. Manuf. 76 (2015) 281-288. doi:10.1016/j.compositesa.2015.04.022.

[5] M. Moevus, N. Godin, M. R'Mili, D. Rouby, P. Reynaud, G. Fantozzi, G. Farizy, Analysis of damage mechanisms and associated acoustic emission in two $\mathrm{SiCf} /[\mathrm{Si}-\mathrm{B}-\mathrm{C}]$ composites exhibiting different tensile behaviours. Part II: Unsupervised acoustic emission data clustering, Compos. Sci. Technol. 68 (2008) 1258-1265. doi:10.1016/j.compscitech.2007.12.002.

[6] C. Simon, F. Rebillat, V. Herb, G. Camus, Monitoring damage evolution of $\mathrm{SiCf} /[\mathrm{SiBC}] \mathrm{m}$ composites using electrical resistivity: Crack density-based electromechanical modeling, Acta Mater. 124 (2017) 579-587. doi:10.1016/j.actamat.2016.11.036.

[7] G.N. Morscher, N.A. Gordon, Acoustic emission and electrical resistance in SiC-based laminate ceramic composites tested under tensile loading, J. Eur. Ceram. Soc. 37 (2017) 3861-3872. doi:10.1016/j.jeurceramsoc.2017.05.003.

[8] E. Maillet, N. Godin, M. R'Mili, P. Reynaud, G. Fantozzi, J. Lamon, Real-time evaluation of energy attenuation: A novel approach to acoustic emission analysis for damage monitoring of ceramic matrix composites, J. Eur. Ceram. Soc. 34 (2014) 1673-1679. doi:10.1016/j.jeurceramsoc.2013.12.041.

[9] A. Morales-Rodriguez, P. Reynaud, G. Fantozzi, J. Adrien, E. Maire, Porosity analysis of long-fiber-reinforced ceramic matrix composites using $X$-ray tomography, Scr. Mater. 60 (2009) 388-390. doi:10.1016/j.scriptamat.2008.11.018.

[10] L. Gélébart, C. Chateau, M. Bornert, J. Crépin, E. Boller, X-ray tomographic characterization of the macroscopic porosity of chemical vapor infiltration $\mathrm{SiC} / \mathrm{SiC}$ composites: Effects on the elastic behavior, Int. J. Appl. Ceram. Technol. 7 (2010) 348-360. doi:10.1111/j.1744-7402.2009.02470.x.

[11] R. Bertrand, O. Caty, V. Mazars, S. Denneulin, P. Weisbecker, J. Pailhes, G. Camus, F. Rebillat, In-situ tensile tests under SEM and X-ray computed microtomography aimed at studying a self-healing matrix composite submitted to different thermomechanical cycles, J. Eur. Ceram. Soc. 37 (2017) 3471-3474. 
doi:10.1016/j.jeurceramsoc.2017.03.067.

[12] C. Chateau, L. Gélébart, M. Bornert, J. Crépin, E. Boller, C. Sauder, W. Ludwig, In situ X-ray microtomography characterization of damage in SiCf/SiC minicomposites, Compos. Sci. Technol. 71 (2011) 916-924. doi:10.1016/j.compscitech.2011.02.008.

[13] H.A. Bale, A. Haboub, A.A. Macdowell, J.R. Nasiatka, D.Y. Parkinson, B.N. Cox, D.B. Marshall, R.O. Ritchie, Real-time quantitative imaging of failure events in materials under load at temperatures above $1,600^{\circ} \mathrm{C}$, Nat. Mater. 12 (2013) 40-46. doi:10.1038/nmat3497.

[14] W. Na, D. Kwon, W.-R. Yu, X-ray computed tomography observation of multiple fiber fracture in unidirectional CFRP under tensile loading, Compos. Struct. 188 (2017) 39-47. doi:10.1016/j.compstruct.2017.12.069.

[15] B.K. Bay, T.S. Smith, D.P. Fyhrie, M. Saad, Digital volume correlation: Threedimensional strain mapping using X-ray tomography, Exp. Mech. 39 (1999) 217-226. doi:10.1007/BF02323555.

[16] L. Saucedo-Mora, M. Mostafavi, D. Khoshkhou, C. Reinhard, R. Atwood, S. Zhao, B. Connolly, T.J. Marrow, Observation and simulation of indentation damage in a SiC-SiCfibreceramic matrix composite, Finite Elem. Anal. Des. 110 (2016) 11-19. doi:10.1016/j.finel.2015.11.003.

[17] M. Mostafavi, N. Baimpas, E. Tarleton, R.C. Atwood, S.A. McDonald, A.M. Korsunsky, T.J. Marrow, Three-dimensional crack observation, quantification and simulation in a quasi-brittle material, Acta Mater. 61 (2013) 6276-6289. doi:10.1016/j.actamat.2013.07.011.

[18] L. Saucedo-Mora, T. Lowe, S. Zhao, P.D. Lee, P.M. Mummery, T.J. Marrow, In situ observation of mechanical damage within a SiC-SiC ceramic matrix composite, J. Nucl. Mater. 481 (2016) 13-23.

doi:10.1016/j.jnucmat.2016.09.007.

[19] F. Bernachy-Barbe, L. Gélébart, M. Bornert, J. Crépin, C. Sauder, Characterization of $\mathrm{SiC} / \mathrm{SiC}$ composites damage mechanisms using Digital Image Correlation at the tow scale, Compos. Part A Appl. Sci. Manuf. 68 (2015) 101-109. doi:10.1016/j.compositesa.2014.09.021.

[20] C. Chateau, T.T. Nguyen, M. Bornert, J. Yvonnet, DVC-based image subtraction to detect microcracking in lightweight concrete, Strain. e12276 (2018).

[21] A. King, N. Guignot, P. Zerbino, E. Boulard, K. Desjardins, M. Bordessoule, N. Leclerq, S. Le, G. Renaud, M. Cerato, M. Bornert, N. Lenoir, S. Delzon, J.P. Perrillat, Y. Legodec, J.P. Itié, Tomography and imaging at the PSICHE beam line of the SOLEIL synchrotron, Rev. Sci. Instrum. 87 (2016). doi:10.1063/1.4961365.

[22] A. Mirone, E. Brun, E. Gouillart, P. Tafforeau, J. Kieffer, The PyHST2 hybrid distributed code for high speed tomographic reconstruction with iterative reconstruction and a priori knowledge capabilities, Nucl. Instruments Methods 
Phys. Res. Sect. B Beam Interact. with Mater. Atoms. 324 (2014) 41-48. doi:http://dx.doi.org/10.1016/j.nimb.2013.09.030.

[23] M. Bornert, J.M. Chaix, P. Doumalin, J.C. Dupré, T. Fournel, D. Jeulin, E. Maire, M. Moreaud, H. Moulinec, Mesure tridimensionnelle de champs cinématiques par imagerie volumique pour l'analyse des matériaux et des structures, Instrumentation, Mes. Métrologie. 4 (2004) 43-88. http://hal.archives-ouvertes.fr/hal-00156072/en/.

[24] Y. Chen, Damage mechanisms in $\mathrm{SiC} / \mathrm{SiC}$ composite tubes: three-dimensional analysis coupling tomography imaging and numerical simulation, $\mathrm{PhD}$ Thesis, Université Paris-Est, 2017.

[25] T.T. Nguyen, J. Yvonnet, M. Bornert, C. Chateau, Initiation and propagation of complex 3D networks of cracks in heterogeneous quasi-brittle materials: Direct comparison between in situ testing-microCT experiments and phase field simulations, J. Mech. Phys. Solids. 95 (2016) 320-350. doi:10.1016/j.jmps.2016.06.004.

[26] A. Lyckegaard, G. Johnson, P. Tafforeau, Correction of ring artifacts in X-ray tomographic images, Int. J. Tomogr. Simul. 18 (2011) 1-9. http://www.ceser.in/ceserp/index.php/ijts/article/view/1164.

[27] T.-T. NGUYEN, Modeling of complex microcracking in cement based materials by combining numerical simulations based on a phase-field method and experimental 3D imaging, 2015.

[28] A.F. Cinar, S.M. Barhli, D. Hollis, M. Flansbjer, R.A. Tomlinson, T.J. Marrow, M. Mostafavi, An autonomous surface discontinuity detection and quantification method by digital image correlation and phase congruency, Opt. Lasers Eng. 96 (2017) 94-106. doi:10.1016/j.optlaseng.2017.04.010.

[29] Y. Chen, L. Gelebart, C. Chateau, M. Bornert, A. King, C. Sauder, P. Aimedieu, Damage initiation and propagation in braided $\mathrm{SiC} / \mathrm{SiC}$ composite tubes: effect of braiding angle, Prep. (n.d.). 